\author{
ARTIFICIAL SATELLITES, Vol. 51, No. 3 - 2016 \\ DOI: 10.1515/arsa-2016-0009
}

\title{
ON THE ANALYSIS OF MULTISTEP-OUT-OF-GRID METHOD FOR CELESTIAL MECHANICS TASKS
}

\author{
L. Olifer ${ }^{1,2}$, V. Choliy ${ }^{3,4}$ \\ ${ }^{1}$ National University "Kyiv Polytechnic Institute", Peremohy ave., 37, 03056, Kyiv, Ukraine \\ ${ }^{2}$ Space Research Institute National Academy of Sciences of Ukraine and State Space Agency of Ukraine, \\ Glushkov Ave 40, 4/1, 03680, Kyiv 187, Ukraine \\ ${ }^{3}$ Taras Shevchenko National University of Kyiv, Glushkova ave., 4, 03127, Kyiv, Ukraine \\ ${ }^{4}$ Main Astronomical Observatory National Academy of Sciences of Ukraine, Zabolotnogo str., 27, 03680, Kyiv \\ e-mail: leonant@ukr.net
}

\begin{abstract}
Occasionally, there is a necessity in high-accurate prediction of celestial body trajectory. The most common way to do that is to solve Kepler's equation analytically or to use Runge-Kutta or Adams integrators to solve equation of motion numerically. For low-orbit satellites, there is a critical need in accounting geopotential and another forces which influence motion. As the result, the right side of equation of motion becomes much bigger, and classical integrators will not be quite effective. On the other hand, there is a multistep-out-of-grid (MOG) method which combines Runge-Kutta and Adams methods. The MOG method is based on using $\mathrm{m}$ on-grid values of the solution and $n \times m$ off-grid derivative estimations. Such method could provide stable integrators of maximum possible order, $O\left(h^{m+m n+n-1}\right)$.

The main subject of this research was to implement and analyze the MOG method for solving satellite equation of motion with taking into account Earth geopotential model (ex. EGM2008 (Pavlis at al., 2008)) and with possibility to add other perturbations such as atmospheric drag or solar radiation pressure. Simulations were made for satellites on low orbit and with various eccentricities (from 0.1 to 0.9 ). Results of the MOG integrator were compared with results of Runge-Kutta and Adams integrators. It was shown that the MOG method has better accuracy than classical ones of the same order and less righthand value estimations when is working on high orders. That gives it some advantage over "classical" methods.
\end{abstract}

Keywords: Out-of-grid methods, numerical integrators.

\section{INTRODUCTION}

Today there are a lot of packages (GMAT ${ }^{1}$ etc.) to solve celestial mechanics tasks. Vital part of them is an integrator and most commonly used - based on Runge-Kutta (RK) or Adams algorithms.

\footnotetext{
${ }^{1}$ https://gmat.gsfc.nasa.gov/
} 
Runge-Kutta algorithms (Fig. 1) propagate a solution over an interval between Oshaped points on Fig. 1 by combining the information from several Euler-style steps (each involving one evaluation of the right-hand side of differential equation (force) on the positions, marked with $\mathrm{X}$ on Fig. 1), and then using the information obtained to match a Taylor series expansion for the polynomials up to the highest possible degree. In other words, this method uses linear combination of force values in different points and adds it to already gained solution to predict one on the next step.

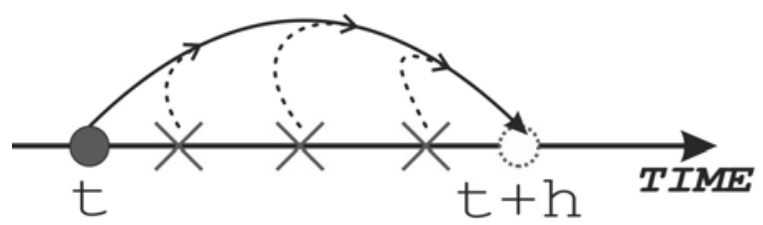

Fig. 1: Geometry of the Runge Kutta method. Points with outgoing dash lines are points, in which the right side of a differential equation (DE) is accounted to predict solution of a DE on the next step; points with the solid lines - points in which the solutions of DE is accounted.

Adams algorithms (Fig. 2), frequently entitled as predictor-corrector multistep methods, store the solution along the way in O-marked positions, and use right-hand sides to extrapolate the solution one step advanced. Then they correct the extrapolation using derivative information at the new point (Press at al., 1992). The approach is the best for very smooth functions and because the orbit of a satellite is quite smooth (the main part of such orbit is an ellipse) - this method is widely used to solve ballistics and celestial mechanics tasks. As one can see, Adams method, analogically to RK, takes linear combination of force values.

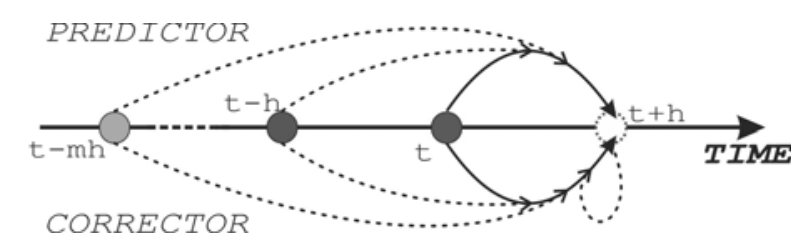

Fig. 2: Geometry of the Adams multistep method. Points with outgoing dash lines are points, in which the right side of a DE is accounted; points with the solid lines - points in which the solutions of a DE is accounted.

Nevertheless, Adams algorithm has some shortcomings: it cannot start by itself and one should manipulate with the step and order to achieve the precision specified. Strictly speaking, those shortcomings are not very critical. There is quite famous VASOMI (VAriable Step and Order Method of Integration) procedure (Taradiy and Tsesis, 1984) where those are solved. Much worse is breaking the Dahlquist theorem by high order Adams algorithms (Dahlquist, 1956). It makes high-order Adams algorithms unstable.

It is obvious that if task requires high accuracy - high order integrators should be used. Because of their nature, the forces would be estimated frequently along all the integration. It is absolutely crucial for low-orbit satellites, for which force is given by the 
equation:

$$
\vec{F}(r)=-\vec{\nabla}\left(-\frac{\mu}{r}+\frac{\mu}{r} \sum_{n=0}^{\infty} \sum_{k=0}^{n}\left(\frac{a_{p}}{r}\right)^{n} P_{n}^{k}(\cos \varphi) \cdot\left(C_{n k} \cos k \lambda+S_{n k} \sin k \lambda\right)\right)+\vec{F}_{\text {other }} .
$$

where $\mu$ - Earth standard gravitational parameter; $a_{p}$ - equatorial radius of the Earth; $P_{n}^{k}$ - associated Legendre polynomials; $C_{n k}$ and $S_{n k}$ - coefficients given by EGM2008; $\vec{F}_{\text {other }}$ - other perturbation forces; $r, \lambda$ and $\varphi$ - coordinates of a satellite in the spherical system.

Frequent evaluation of forces requires a lot of resources and time. The integrator with as little as possible right hand side estimations giving the best precision is definitely the best one.

\section{MULTISTEP-OUT-OF-GRID METHOD}

\section{DESCRIPTION}

In general, Multistep-out-of-grid (MOG) method could be presented as a combination (hybrid) of the RK and Adams methods for numerical integrating of ordinary differential equation. The MOG algorithm behaves as the RK one when estimating off-grid values and like Adams predictor-corrector when estimating on-grid values. The method was introduced and analyzed in (Beaudet, 1972). There are no articles on the MOG algorithm known to the authors.

Numerical solution of the system of ordinary differential equation of the first order:

$$
\frac{d \vec{Y}}{d t}=F(\vec{Y}, t) ; \quad \vec{Y}(0)=\vec{Y}_{0}
$$

with the MOG algorithm is based on using $m$ on-grid values of the solution, which were gained on the previous steps (equivalent to Adams method) and $n$ off-grid derivative values estimated on previous steps, for each on-grid element (just like in the Runge-Kutta method - between two solutions we have points with derivative values). Together this method is using $m$ on-grid values of the solution and $n m$ off-grid derivative values. (Fig. 3). As one can see, here is used linear combination of force values and linear combination of already gained solutions from different points.

Combining them we could write down the following formula for calculating on-grid scalar value which is called refiner:

$$
Y_{N+1}=\sum_{j=0}^{m-1} \alpha_{i} Y_{N-j}+h \sum_{j=0}^{m-1} \sum_{l=1}^{n} \beta_{l, j} F_{N-j+\gamma_{l}}
$$

where $\alpha_{i}$ and $\beta_{l, j}$ - are the weighting coefficients and $h \cdot \gamma_{l}$ - is the distance from corresponding on-grid element to $l$-s off-grid one on the same step along time axis. Value of $n+m+n m$ is a number of unknowns in (2). The value $n+m+n m-1$ specifies the method order, which is the maximum degree of polinomial for which the refiner produces no errors. 


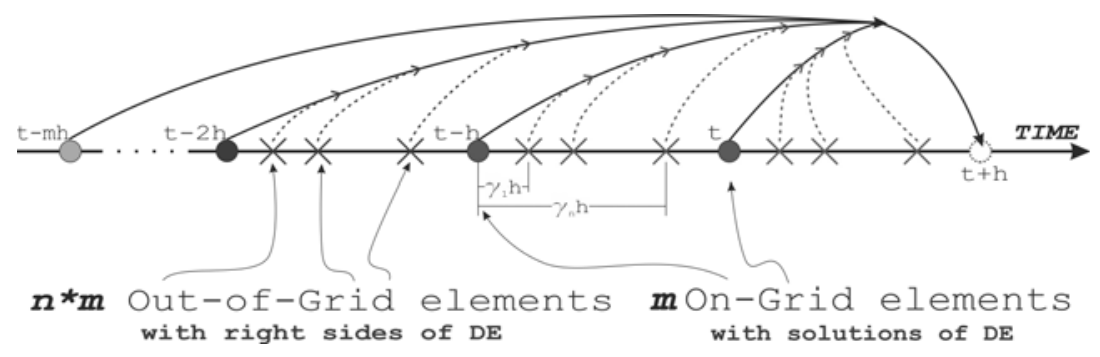

Fig. 3: Geometry of the Multistep-out-of-grid method. Points with outgoing dash lines are points, in which the right side of a DE is accounted; points with the solid lines points in which the solutions of a DE is accounted.

Analyzing geometry of the integrator we already could say that the MOG has advantage over classical methods because for the same order of error the MOG uses fewer previously estimated values than Adams method and has fewer number of calculations than the Runge-Kutta algorithm.

To find solution on the $l^{\prime}$ off-grid element there is special formula:

$$
Y_{N+\gamma_{l^{\prime}}}=Y_{N}+h \sum_{j=0}^{k} \sum_{l=1}^{n} b_{l, j}\left(\gamma_{l^{\prime}}\right) F_{N-j+\gamma_{l}}
$$

where $b_{l, j}$ - the coefficients to be determined. Like in Adams algorithm, every $Y_{N+\gamma_{l^{\prime}}}$ should be predicted first and then corrected. The used formula are the same, but for predictor, the value of $b_{l^{\prime}, j}$ is equal to zero. $k$-summation parameter, selected to have amount of different $b_{*}$ equal to the method order.

In general, for every $\gamma$ the predictor is used once then as much of correctors as necessary to achieve the desired precision. That is why the MOG may be represented as Predict-Estimate-Correct-Estimate algorithm with $z$ number of Correct-Estimate step $\left(P E(C E)^{z}\right)$. In most cases PECE is absolutely sufficient.

\section{Coefficients of the MOG Formulae}

To derive system of equations for $\alpha, \beta, \gamma$ the refiner formula (2) is expanded in a Taylor series about any convenient point. Typically point $N$ is selected. The result is:

$$
\sum_{i=0}^{\infty} Y_{N}^{(i)} \frac{h^{i}}{i !}=\sum_{j=0}^{m-1} \alpha_{i} \sum_{i=0}^{\infty} Y_{N}^{(i)} \frac{(-j h)^{i}}{i !}+h \sum_{j=0}^{m-1} \sum_{l=1}^{n} \beta_{l, j} \sum_{i=0}^{\infty} F_{N}^{(i)} \frac{\left[\left(\gamma_{l}-j\right) h\right]^{i}}{i !}
$$

Then, replace $F_{N}^{(i)}$ with $Y_{N}^{(i+1)}$ and require that coefficients of $Y_{N}^{(i)} \frac{h^{i}}{i !}$ to be zero for all $i$-s, gives:

$$
1=\sum_{j=0}^{m-1} \alpha_{i}(-j)^{i}+i \sum_{j=0}^{m-1} \sum_{l=1}^{n} \beta_{l, j}\left(\gamma_{l}-j\right)^{i-1} .
$$

Same logic used for $b_{l, j}$ coefficients and equation (3) gives system of linear equations for $i=1, n+m+n m-1$ :

$$
\gamma_{l^{\prime}}=\sum_{j=0}^{k} \sum_{l=1}^{n} b_{l j}\left(\gamma_{l}-j\right)^{i-1}
$$


This system could be solved using Gauss method.

The equation (4) could be solved using Newton method. It was shown that variation of initial conditions for $\gamma$ lead to different stable solutions for each topology $(m, n)$ and as the result to different solutions for $b(\gamma)$. As an example, graphical representation of obtained solutions for topology $(2,3)$ is shown on (Fig. 4).

As one can see $\gamma$-s could be greater than one. In this case, the predictor extrapolates, corrector interpolates, but refiner become interpolator (not extrapolator as it follows from 2) and it definitely is more accurate than extrapolator. Still, such assumption has to be investigated.

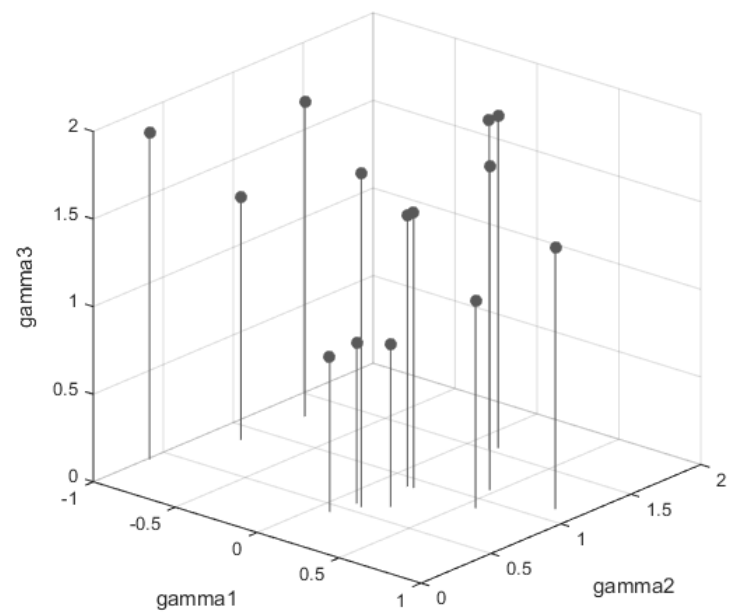

Fig. 4: Different $\gamma$ coefficients for the same topology $(2,3)$.

\section{RESULTS}

To check the MOG it is better to start with solving Kepler's problem $\ddot{\vec{r}}=-\frac{\mu}{r^{3}} \vec{r}$ for different satellites. Such equation has exact solution thus it will be easier to check accuracy of the integrator.

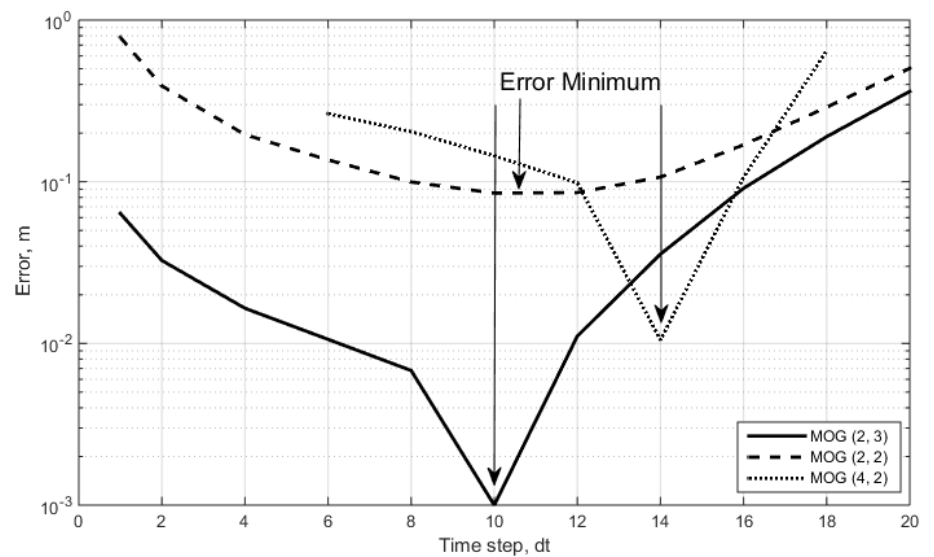

Fig. 5: Relation between time step and errors for different topoloies. 
Table 1: Results of simulations for different MOG(2,3) coefficient sets.

\begin{tabular}{|c|c|c|}
\hline Gammas & Error, $\mathrm{m}$ & Integration time, $\mathrm{s}$ \\
\hline $0.181 ; 0.905 ; 1.575$ & 0.011 & 1015 \\
$0.148 ; 1.547 ; 1.897$ & 0.012 & 1038 \\
$0.469 ; 1.112 ; 1.846$ & 0.040 & 1013 \\
$0.149 ; 0.899 ; 1.550$ & 0.048 & 1012 \\
$0.568 ; 0.895 ; 1.187$ & 0.066 & 1011 \\
$-0.829 ; 0.854 ; 1.385$ & 0.143 & 1084 \\
$0.110 ; 0.581 ; 0.914$ & 0.144 & 1013 \\
$0.852 ; 1.132 ; 1.488$ & 0.215 & 1013 \\
$0.258 ; 0.651 ; 0.928$ & 0.278 & 1012 \\
$-0.797 ; 1.274 ; 1.793$ & 0.323 & 1328 \\
$0.128 ; 1.508 ; 1.880$ & 0.550 & 1066 \\
\hline$-0.987 ; 0.386 ; 1.862$ & Solution does not converge \\
$0.155 ; 0.561 ; 1.904$ & \multicolumn{2}{|c|}{ Solution does not converge } \\
\hline
\end{tabular}

For analyzing different coefficient sets which were obtained for topology $(2,3)$ such initial conditions were chosen: position $-\vec{r}=(6380,0,0) \mathrm{km}$, eccentricity $-e=0.1$, time step $-d t=6$ sec. Simulations were made for 200 revolutions. Results of which are shown at Table 1 . the error was estimated as difference between simulated orbit and analytical solution on the end of $200^{\text {th }}$ revolution.

As one can see the MOG can provide a nice accuracy. However, to find the reason why different coefficient sets give different errors further studies are needed.

Property which also has to be investigated is how accuracy depends on time step. Simulations with the same initial conditions were made for topologies $(2,2),(2,3),(4,2)$. Results are shown in Figure 5.

Table 2: Results of simulations for different integrators

\begin{tabular}{|c|c|c|c|}
\hline method & MOG $(2,3)$ & Runge Kutta & Adams PECE \\
\hline order & 10 & 4 & 10 \\
\hline time step & $10 \mathrm{sec}$ & $10 \mathrm{sec}$ & $1 \mathrm{sec}$ \\
\hline eccentricity & \multicolumn{3}{|c|}{ Error } \\
\hline 0.1 & $0.13 \mathrm{~m}$ & $23 \mathrm{~m}$ & $0.81 \mathrm{~m}$ \\
0.5 & $0.9 \mathrm{~m}$ & $102 \mathrm{~m}$ & $1.25 \mathrm{~m}$ \\
0.9 & $120 \mathrm{~m}$ & $8780 \mathrm{~m}$ & $156 \mathrm{~m}$ \\
\hline eccentricity & \multicolumn{3}{|c|}{ Right sides estimations } \\
\hline 0.1 & 356389 & 475200 & 1187964 \\
0.5 & 860674 & 1147576 & 2868912 \\
0.9 & 9622616 & 12830168 & 32075388 \\
\hline eccentricity & \multicolumn{3}{|c|}{ Time of integration } \\
\hline 0.1 & $608 \mathrm{sec}$ & $22 \mathrm{sec}$ & $234 \mathrm{sec}$ \\
0.5 & $1570 \mathrm{sec}$ & $52 \mathrm{sec}$ & $857 \mathrm{sec}$ \\
0.9 & $20571 \mathrm{sec}$ & $605 \mathrm{sec}$ & $8765 \mathrm{sec}$ \\
\hline
\end{tabular}


For each method there is an optimal time step where the error is the smallest. Additionally the MOG has an interesting property: After increasing order (from $(2,2)$ to $(4,2))$ we also could increase the time step and still get better accuracy. It does not work for classical multistep methods and is a nice way to continue our research and find an appropriate proof the Dahlquist theorem violation for the MOG.

Comparison results of $(2,3)$ MOG integrator with classical ones (Table 2) shows that the MOG accuracy is better and number of force evaluations is lower than in the RK and Adams methods. Here, "time of integration" mostly shows time which were spent for integrator realization, not for the force estimation (because for Kepler problem case force equation is relatively small). Thus, experiments with larger force equations (accounting geopotential) could be made faster using the MOG, than classical methods for the same accuracy.

\section{CONCLUSIONS}

1. Multistep Out-of-Grid Method was implemented to solve Kepler problem for satellites with different eccentricities for 200 revolutions.

2. Accuracy depends on eccentricities (not really surprising). Still, it is much higher for equivalent classical Runge-Kutta or Adams methods.

3. For the same order (topology) different method coefficients could be obtained. All of them could show different result. Such appearance could be depended to the simulating system nature.

4. For the MOG we could find Dahlquist theorem violation which means that order of method and time step could be increased simultaneously.

\section{REFERENCES}

Beaudet P. (1972) Multi-off-grid methods in multi-step integration of ordinary differential equations, in Proc. of the Conference on the Numerical Solutions of ODE, 1972, Texas Univ., USA, 498 p.

Dahlquist G. (1956) Convergence and stability in the numerical integration of ODE, Math.Scand., Vol. 4, 33-53

Pavlis N., Holmes S., Kenyon S., Factor J. (2008) An Earth Gravitational Model to Degree 2160: EGM2008, Presented at the 2008 General Assembly of the European Geosciences Union, Vienna, Austria, April 13-18, 2008.

Press W., Teukolsky S., Vetterling W., Flannery B. (1992) Numerical recipes in C, Cambridge.: Cambridge University Press, 1992.

Taradiy V., Tsesis M. (1984) Computation of the Earth' satellites trajectories. Implementing of the Adams algorithm and program of variable step and order. Preprint / AS UkrSSR. Institute of theoretical physics N ITF-84-92P,- in Russian., Kyiv, 1984.

Received: 2016-05-31,

Reviewed: 2016-07-01, by V.V. Pashkevich, and 2016-09-05, Accepted: 2016-09-05. 